\title{
CASES OF EPIDEMIC CEREBROSPINAL MENINGITIS TREATED WITH FLEXNER'S ANTIMENIN- GITIS SERUM
}

\author{
WILLIAM ALEXANDER SMITH, M.D. \\ WEBSTER GROVES, MO.
}

The following cases are placed on record because they show (1) the value of Flexner's antimeningitis serum in cases of epidemic cerebrospinal meningitis, even after the infection has lasted for many days or weeks; (2) the necessity for prolonged treatment in such cases, and on the other hand (3) the rapidity of cure when the serum is injected promptly after the manifestation of symptoms.

The first three cases reported were in children of one family.

CASE 1.-I. G., aged 8 months, was admitted to the hospital April 25, 1912. He was the yourngest of four children. The onset of his present illness was April 16,1912, with fever and irritability. When he was picked up he screamed with pain. After a continuation of these symptoms for five days, it was observed that he did not use his right arm. He remained fretful, his paralysis and fever continued, and for these reasons he was brought to the hospital, but chiefly because his sister, who was taken ill after him with a much more severe form of the disease, had already been admitted and had begun to show improvement from treatment. On examination in the ward (April 25, 1912), there was extreme hyperesthesia and he was exceedingly irritable. There was a paresis of the right arm, a tache cérébrale and exaggerated reflexes at the kneeKernig's sign was absent, the neck was not rigid and the anterior fontanel not bulging - the temperature was $102.5 \mathrm{~F}$. The leukocytes were 34,000 .

Lumbar puncture No. 1 was done shortly after admission. Thirty c.c. of turbid fiuid were removed and 20 c.c. of Flexner's serum were injected. The fluid was turbid and under increased pressure. The cells were mainly polymorphonuclear leukocytes and contained Gram-negative diplococci. The meningococcus was obtained from the fluid in culture. The Noguchi globulin reaction was positive. No unfavorable symptoms followed the injection of the serum.

The next morning (April 26, 1912) the temperature was $99 \mathrm{~F}$., but as there was no other appreciable improvement in the child's condition, lumbar puncture No. 2 was done; 20 c.c. of fluid were removed and 20 c.c. of serum were injected. The fluid was cloudy. There were many pus cells but no organisms were found. Culture showed no growth. After this injection the temperature rose to $105 \mathrm{~F}$., but in twelve hours had fallen to $99 \mathrm{~F}$.

The following day (April 27, 1912), as there was still no improvement in the general condition, lumbar puncture No. 3 was done; 60 c.c. of fluid were removed and 15 c.c. of serum injected. The fluid was clear. There were very few pus cells and no organisms were found. There was a temperature reaction to $104 \mathrm{~F}$. following this injection. The child showed improvement in the next twenty-four hours by being less irritable and less hyperesthetic. The right arm was still helpless, but the reflexes at the knee were not exaggerated. Lumbar puncture No. 4 was done on this day (April 28, 1912); 15 c.c. of fluid were removed and 15 c.c. of serum injected. The fluid was clear and contained no pus cells or organisms. The temperature rose to $103 \mathrm{~F}$. after this injection. The leukocytes were 15,000 . 
Lumbar punctures Nos. 5, 6 and 7 were done on the next three days and 20,30 and 50 c.c. of clear fluid, respectively, withdrawn, containing no pus cells and no organisms. The punctures were performed in order to see if organisms were present, in which case serum was to be again injected, but also because each time, with a considerable accumulation of fluid, there was restlessness and irritability. These symptoms were at once relieved by withdrawing the excess of fluid. The child's improvement was marked, but as the temperature rose to $102 \mathrm{~F}$. on May 2, 1912, lumbar puncture No. 8 was done and 20 c.c. of fluid were obtained. The fluid was cloudy and contained many pus cells. For this reason 20 c.c. of serum were injected, but no organisms were found and the

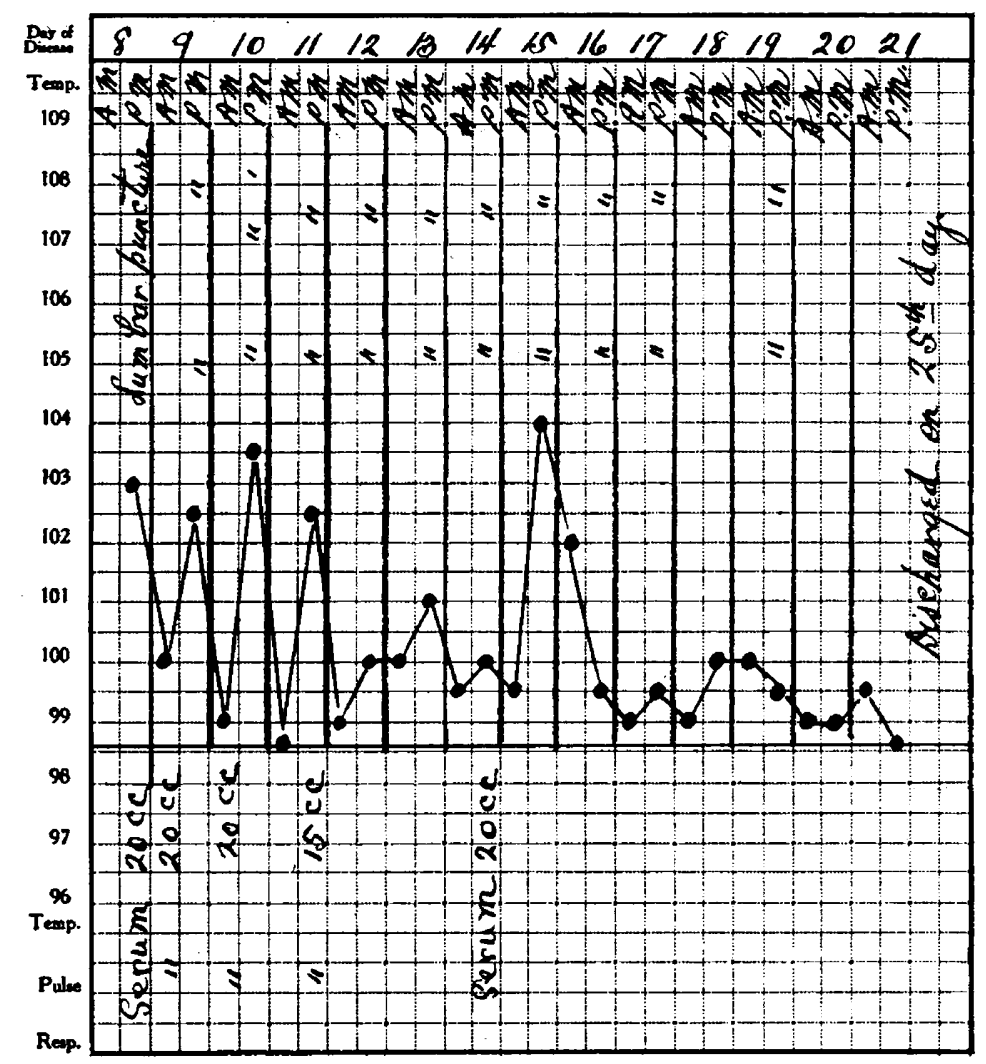

Chart 1.-Temperature curve in Case 1.

cultures were negative. The leukocytes were 24,000 . The temperature rose to $104 \mathrm{~F}$. after the injection. Lumbar punctures Nos. 9 and 10 made on the following two days showed slightly cloudy fluid with few pus cells, but no organisms. The Noguchi globulin reaction was still positive.

Lumbar puncture No. 11 was made two days later (May 6, 1912); 10 c.c. of fuid were obtained. This was clear. There were no pus cells and the Noguchi reaction was negative. The leukocytes were 12,000 .

From this time the child improved rapidly. The temperature remained normal and the power in the right arm returned. He was discharged May 11, 1912, entirely well. 
In this case the hyperesthesia, marked irritability of the child and the paresis of the right arm, were strongly suggestive of meningitis even in the absence of rigidity of the neck, Kernig's sign and bulging of the fontanel. It is interesting to observe that four days after the last injection of serum the spinal fluid became cloudy and that there were many pus cells but no organisms.

The reactions due to the serum were definite and sharp but were of short duration and entirely without sequelae.

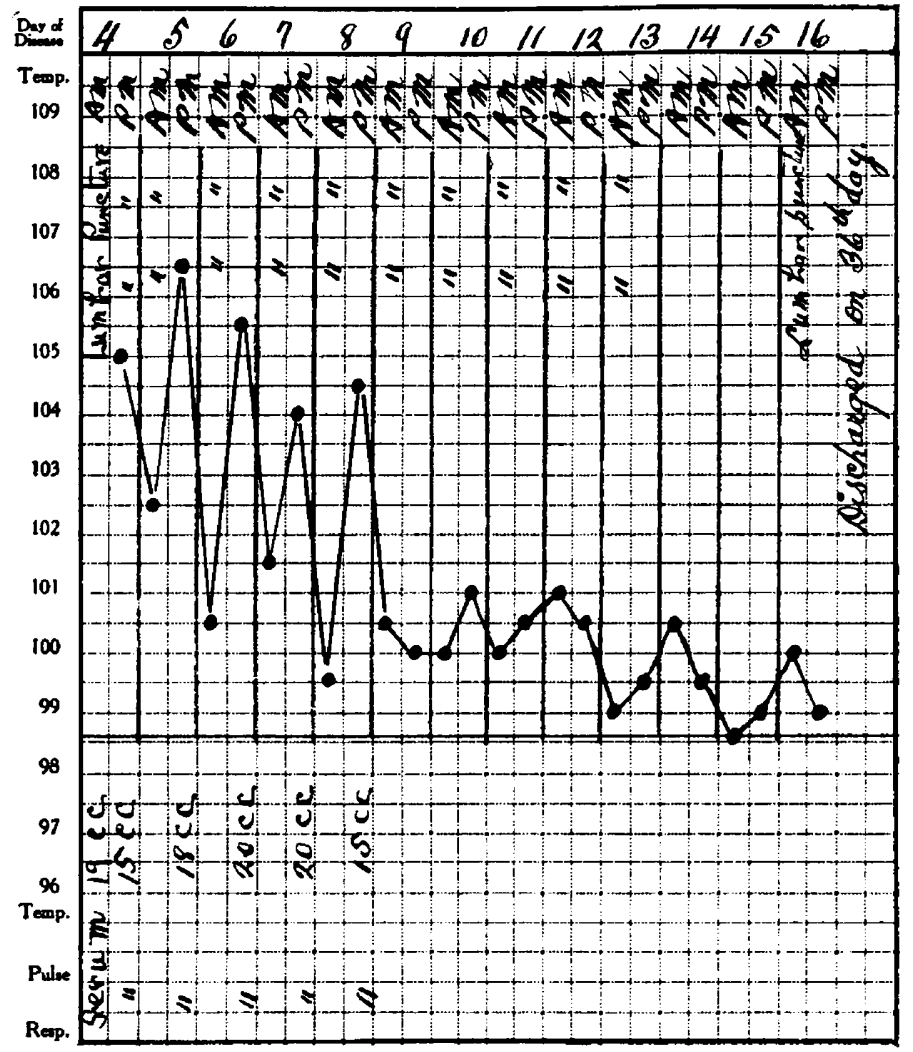

Chart 2.-Temperature curve in Case 2.

CASE 2.-F. G., aged 21/2 years, was admitted to the hospital April 24, 1912. The onset of the present illness began April 20, 1912, four days after that of her brother (Case 1). She was taken acutely ill with fever, vomiting, headache in the left frontal region and convulsions. The next day (April 21, 1912) there was in addition a petechial eruption about the face and chest. Her condition remained the same during the next two days and on admission to the ward, April 24, 1912, she was stuporous and having one convulsion after another. There was slight rigidity of the neck muscles, internal strabismus of the right eye, a coarse tremor of the extremities, exaggerated reflexes, a tache cérébrale, a bilateral Babinski and a positive Macewen's sign. Kernig's sign was not present. The temperature was $105 \mathrm{~F}$; the leukocytes 28,000 . 
Lumbar puncture No. 1 was done at once; 20 c.c. of thick purulent fluid were withdrawn and 15 c.c. of Flexner's serum injected. The fluid showed many pus cells and Gram-negative intracellular diplococci. The meningococcus was obtained by culture. The Noguchi globulin reaction was positive. There was a severe reaction after the injection of the serum, as shown by the rise of temperature and an increase of the nervous symptoms. This lasted about one hour.

The child's general condition improved after the first injection and the next day, April 25, 1912, she could be aroused, the temperature was $101 \mathrm{~F}$. and there were no convulsions.

Lumbar puncture No. 2 was done; 35 c.c. of fluid were withdrawn and 20 c.c. of serum injected. The fluid was turbid and contained many pus cells, but few organisms. The cultures were positive. After this injection of serum there was a severe reaction. The temperature rose to $107 \mathrm{~F}$., the respiration was irregular and rapid and there was a coarse general tremor. No convulsions.

On the following day (April 26, 1912) the temperature was $100 \mathrm{~F}$., and although she lay for the most part in a stupor she recognized her parents when aroused. The reflexes were not exaggerated and there were no convulsions. Lumbar puncture No. 3 was done; 35 c.c. of fluid were removed and 20 c.c. of serum injected. The fluid was cloudy. There were many pus cells but only a few organisms. The organisms were extracellular. The cultures showed no growth. The Noguchi reaction was positive. There was the same severe reaction lasting for about one hour after this injection.

April 27, 1912, although the child was in every respect improved, Lumbar puncture No. 4 was done; 35 c.c. of fluid were obtained and 20 c.c. of serum injected. The fluid was clear, containing only a few pus cells and no organisms. The Noguchi reaction was positive. The reaction after this injection was not severe except for the high temperature.

Lumbar puncture No. 5 was done April 28, 1912; 20 c.c. of clear fluid containing no organisms were obtained; 15 c.c. of serum were injected. The Noguchi reaction was negative. Lumbar puncture was done the five days following. Twenty to 70 c.c. of clear fluid containing no pus cells or organisms were obtained. After an interval of two days 80 c.c. of clear fluid were obtained by lumbar puncture No. 11. This fluid showed no pus cells or organisms. The Noguchi reaction was negative. The indications for the repeated withdrawal of fluid were the same as with the first child. The child improved rapidly after the fifth injection of serum. The temperature varied between $99 \mathrm{~F}$. and $101 \mathrm{~F}$., gradually becoming normal. The leukocytes fell to 12,000 and except for a serum rash ten days after the first injection of serum, the convalescence was uneventful. During convalescence lumbar punctures, four in all, were done at intervals. Each time from 10 to 30 c.c. of clear fluid were obtained.

The case was a severe one. Four days after the onset the child was in a stupor, the temperature was very high and she was having frequent convulsions.

CASE 3.-M. G., aged 6 years, was taken ill April 26, 1912, at noon, ten days after her brother (Case 1). The onset was acute, with vomiting, frontal headache and fever. She was admitted to the hospital four hours later $(4 \mathrm{p} . \mathrm{m}$., April 26, 1912) after having had several convulsions. On admission the temperature was $103 \mathrm{~F}$., there was drowsiness and she complained, when aroused, of intense headache. On examination there was a tache cérébrale, slightly exaggerated reflexes and dilated pupils. Macewen's sign was present. There was no rigidity of the neck muscles, nor was Kernig's sign present. The leukocytes were 30,000 .

Lumbar puncture No. 1 was done at once; 25 c.c. of fluid were withdrawn and 20 c.c. of Flexner's serum injected. The fluid was clear but contained, after centrifugation, a few pus cells and Gram-negative intracellular diplococci. The cultures showed meningococci. The Noguchi globulin reaction was positive. 
The following day (April 27, 1912) there was a fine petechial eruption on the upper part of the chest and neck. The patella tendon reflexes were markedly exaggerated and Kernig's sign was present. In other respects, however, there had been a marked improvement. The temperature was lower (100 F.) and the child was much brighter and complained no longer of headache. Lumbar puncture was not done. From this day improvement was rapid. The temperature remained normal and the number of leukocytes fell to 12,000 (May 7, 1912). Convalescence was uneventful except for the appearance of an urticarial eruption on the seventh day and the child was discharged May 7, 1912 , eleven days after the onset of the disease.

These three cases show almost with the nicety of a carefully planned experiment the value of the serum treatment and the rapidity of its effect in direct proportion to the time of its employment. Children

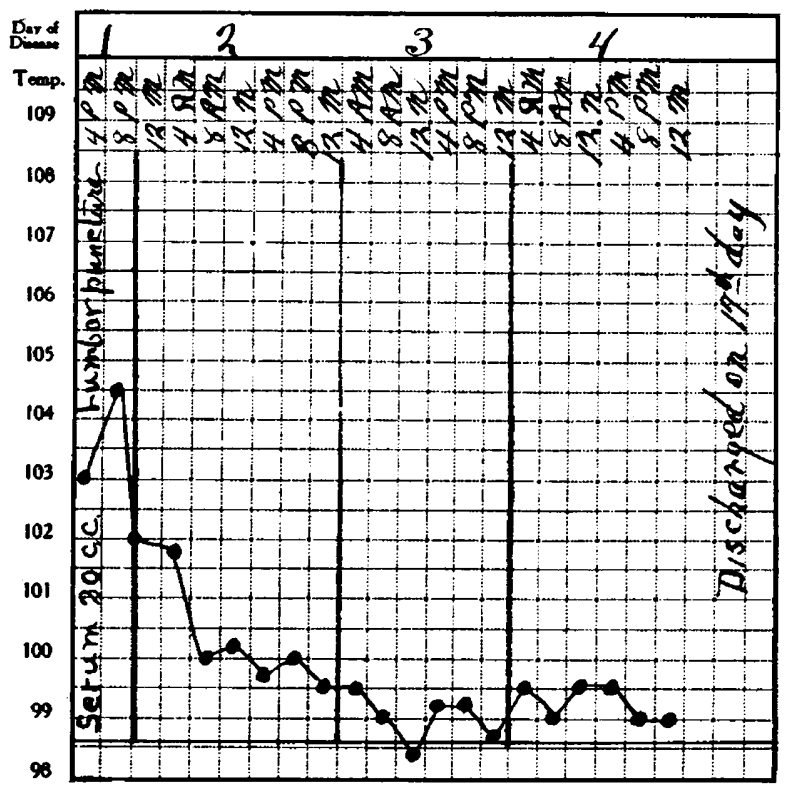

Chart 3.-Temperature curve in Case 3.

1 and 2 had been ill nine and four days, respectively, before the institution of treatment. Both were severely ill, the second child particularly so. In both the disease yielded to treatment, but only after many days and repeated injections of the serum. In marked contrast is the case of Child 3, a member of the same family. Her disease was marked by a sudden and severe onset, yet it yielded promptly and permanently to only one injection of the serum. I know of no similar recorded case in which treatment was begun so soon (four hours) after the beginning of symptoms. The temperature charts show graphically this distinction. Of particular interest was the fact that the duration of the disease was so short that the cerebrospinal fluid had not yet 
hecome turbid. Such a fluid though unusual is occasionally encountered. The presence of meningococci, together with the perfectly characteristic nervous symptoms and the petechial eruption, leave no doubt of the diagnosis. Unless other children in the same family had previously suffered from similar symptoms, it is likely that hours or days would have elapsed before serum treatment would have been possible.

The two following cases are examples of prolonged meningococcus meningitis showing no tendency to improve, but yielding at once to the serum:

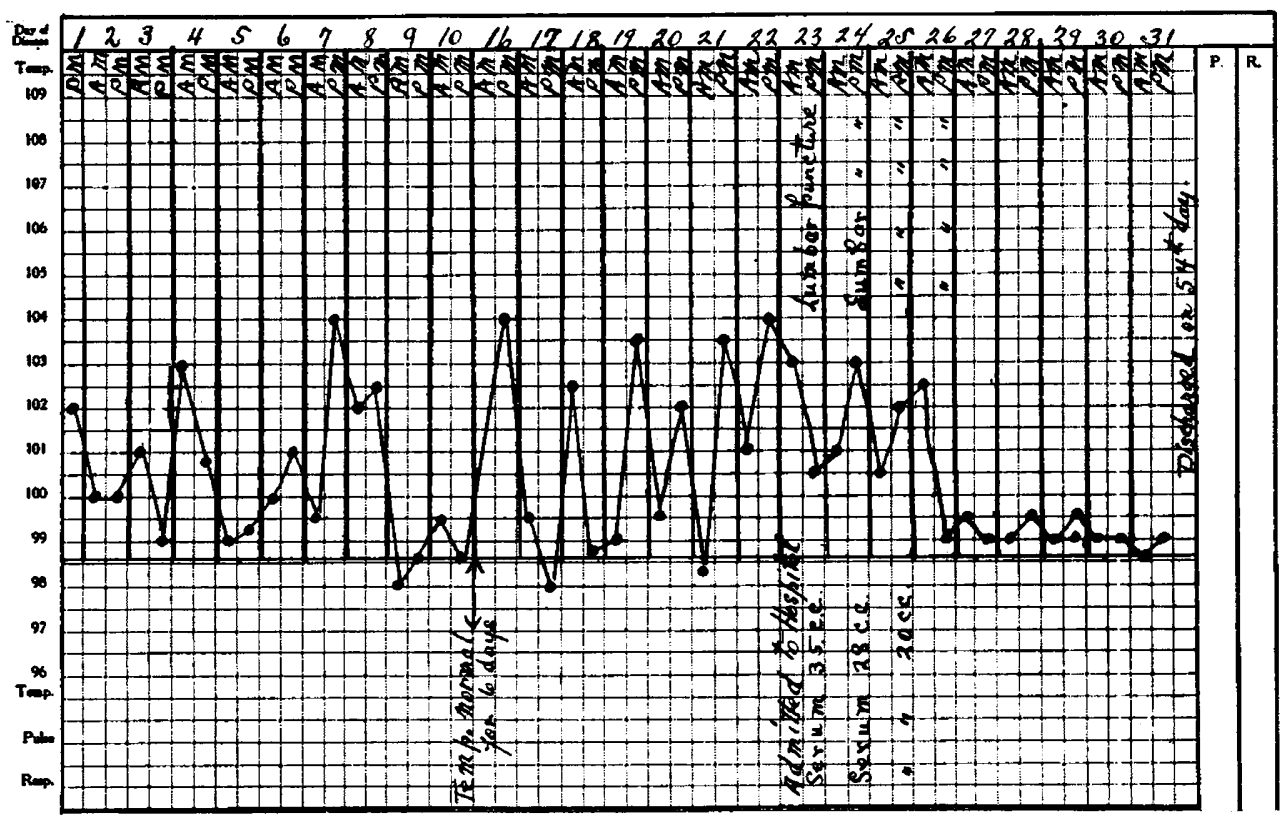

Chart 4.-Temperature curve in Case 4.

CASE 4.-L. A., aged 4 years, was admitted to the hospital May 23, 1912. The present illness began April 30, 1912, with fever, convulsions and headache. The temperature varied intermittently from 97 to $104 \mathrm{~F}$. and the convulsions continued at irregular intervals until May 9, 1912. Then the temperature remained normal and he was up and about the house until May 15, 1912, a period of six days. On the fifteenth he had a convulsion and the temperature rose to $104 \mathrm{~F}$. The intermittent temperature and convulsions continued and he was admitted to the hospital May 23, 1912, twenty-three days after the onset of the disease.

On examination in the ward he was found to be greatly prostrated, emaciated, irritable and complained of a severe headache. There was marked hyperesthesia, rigidity of the neck, exaggerated reflexes, ankle clonus and a positive Kernig and Macewen sign. At night he had a shrill meningeal cry; the temperature was $103 \mathrm{~F}$. The leukocytes were 24,000. Lumbar puncture No. 1 was done; 25 c.c. of fluid were withdrawn. The fluid was cloudy and 
contained pus cells and many Gram-negative intracellular diplococci. Cultures showed meningococci. The Noguchi globulin reaction was positive; 25 c.c. of Flexner's serum were injected six hours later. During the injection of the serum the child complained of severe pain in the legs and the temperature which was $97 \mathrm{~F}$. afterwards rose to $103 \mathrm{~F}$.

The next day, May 24, 1912, he showed improvement by being less hyperesthetic and less irritable. The temperature remained high. Lumbar Puncture No. 2 was done; 50 c.c. of fluid were removed and 40 c.c. of serum were injected. The fluid was slightly cloudy and contained few pus cells, but no organisms. The Noguchi reaction was positive.

Lumbar puncture No. 3 was done May 25, 1912; 25 c.c. of fluid were withdrawn and 20 c.c. of serum injected. There was a reaction in the temperature to $103 \mathrm{~F}$. The fluid removed at this time was collected in four test tubes. It flowed freely from the needle, and though clear, had a peculiar yellow hue. It was taken immediately, after the serum had been injected, to the laboratory, and there was found to have coagulated to the consistency of solid agar. There were no pus cells nor organisms. The cultures showed no growth.

Lumbar puncture No. 4 was done the next day, May 26, 1912. The fluid appeared in drops at the end of the needle and coagulated at once, and when a drop was wiped off another would appear and rapidly become coagulated. At this time the child showed marked improvement, the temperature remained normal, and except for a great weakness which continued to the time of his discharge (June 23,1912 ) he made an uneventful recovery.

CASE 5.-E. T., aged 6 years, was admitted to the hospital Nov. 27, 1912. The present illness began three weeks previously (Nov. 7, 1912) when she complained of headache and feeling tired. She was in this state for about one week or until Nov. 15, 1912, when she had three general convulsions, each lasting about five minutes. After that she remained in bed with headache and an afternoon rise of temperature to $104 \mathrm{~F}$. One week after this (Nov. 20, 1912) her neck became stiff. Since then she had had headache, afternoon fever and stiffness of the neck. She had not vomited. On admission to the ward she was conscious and complained of headache. There was extreme emaciation, the head was drawn back and the back arched. Macewen's sign was not present. The eye grounds were normal. The abdomen was boat-shaped. There was a bilateral Kernig, exaggerated reflexes and ankle clonus. The temperature was $100 \mathrm{~F}$., the leukocytes 30,400 .

Lumbar puncture No. 1 was done; 20 c.c. of fluid were removed and 20 c.c. of Flexner's serum injected. The serum was turbid and under increased pressure. There were many pus cells and Gram-negative intracellular diplococci and the Noguchi reaction was positive. The meningococcus was obtained by culture. After the injection the temperature rose to $104 \mathrm{~F}$. The next day (Nov. 28, 1912) there was no improvement and lumbar puncture No. 2 was done; 15 c.c. of fluid were removed and 20 c.c. of serum injected. The fluid was turbid and there were a few pus cells with a few Gram-negative intracellular diplococci. Cultures showed the meningococcus. The temperature fluctuated for the next few days from $99 \mathrm{~F}$. to $104 \mathrm{~F}$., but there was no improvement in the child's condition. Lumbar punctures Nos. 3, 4 and 5 were done and 20,40 and 15 c.c., respectively, of serum were injected. There was no growth on cultures and the fluid was clear after the second injection of the serum. The Noguchi reaction remained positive. After the fifth injection, Dec. 1, 1912, the temperature remained normal. The child's general condition was improved and she could move her neck freely from side to side. The retraction continued but was less marked. Dec. 5, 1912, the temperature rose to $103 \mathrm{~F}$., and though she seemed in as good condition as before, lumbar puncture No. 6 was done; 30 c.c. of fiuid were removed and 30 c.c. of serum injected. The fluid was clear; there were no pus cells or organisms. The cultures were 
negative and the Noguchi reaction was negative. The rigidity of the neck became less and less, disappearing about Dec. 11, 1912. Convalescence was slow and she was discharged Dec. 26, 1912.

\section{DISCUSSION}

These five cases varying in duration from four hours to twenty-three days were all favorably influenced by the antimeningitis serum. All the patients recovered without sequelae. A study of these prolonged

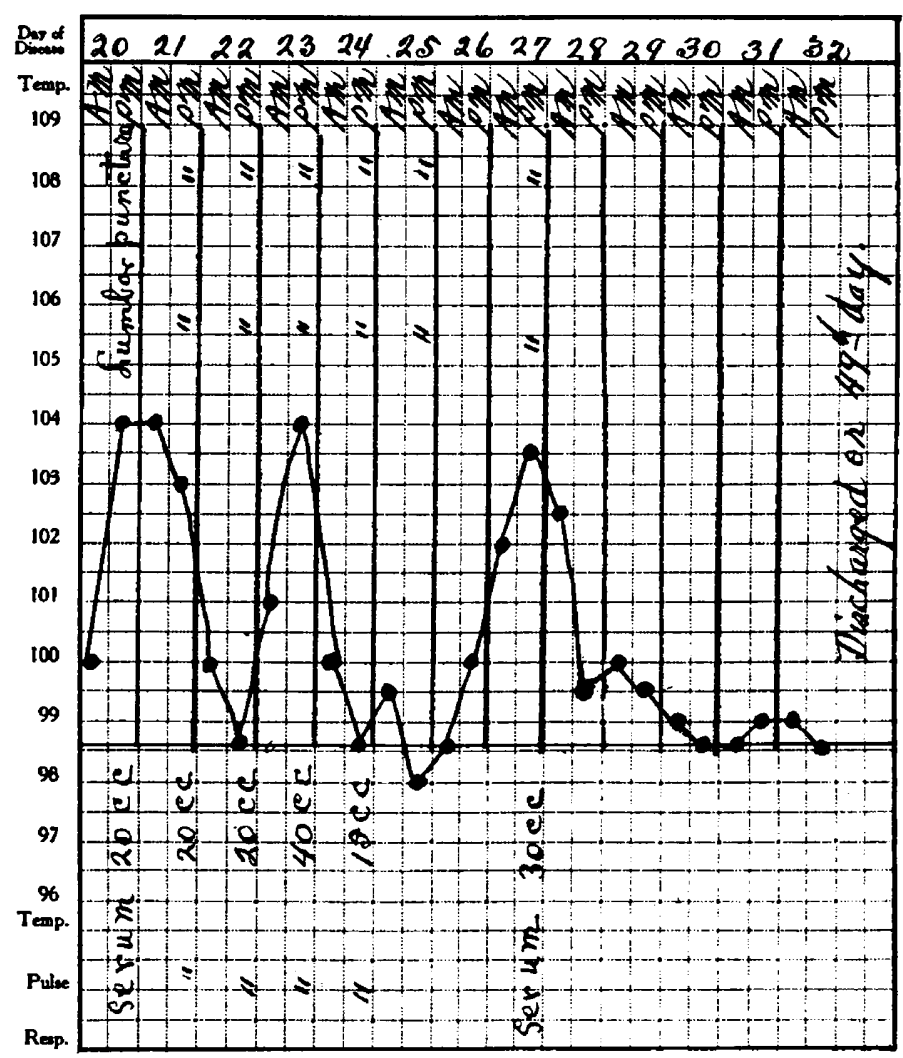

Chart 5.-Temperature curve in Case 5.

cases shows the necessity of repeated injections of the serum and of continuing the withdrawal of cerebrospinal fluid at frequent intervals well after the fluid is clear and no organisms are to be found microscopically or by culture. The nervous symptoms and their prompt amelioration after lumbar puncture make this clear. That such large quantities as 20 to 80 c.c. of fluid can be withdrawn shows that temporarily the injury to the meninges prevents the absorption of the fluid which is undoubtedly formed in excess owing to inflammatory irritation of the choroid plexus. 
Occasionally the fluid may exhibit unexpected and peculiar characteristics, as in Case 4, in which spontaneous coagulation took place, and in Case 1, in which in convalescence a cloudy fluid due to polymorphonuclear leukocytes appeared without any cocci or other organisms being present and following several findings of perfectly clear fluid.

The reactions due to the repeated injections of foreign serum were marked. There was frequently high fever and restlessness, but these were of short duration.

These cases while few in number serve nevertheless to emphasize the fact that in any case of meningococcus meningitis, no matter of what duration, provided viable meningococci are present, Flexner's antimeningitis serum should be used repeatedly, and that even in apparently unfavorable cases, complete recovery may occur.

The first four cases were observed in the St. Louis Children's Hospital in the service of Dr. Howland, and I am further indebted to him for the opportunity of reporting the fifth case.

122 West Lockwood Avenue. 